\title{
Behavior, Displacement and Pregnancy Loss in Pigs under an Electronic Sow Feeder
}

\author{
Ryosuke Iida $^{1}$, Carlos Piñeiro ${ }^{2} \&$ Yuzo Koketsu ${ }^{1}$ \\ ${ }^{1}$ School of Agriculture, Meiji University, Kanagawa, Japan \\ ${ }^{2}$ PigCHAMP Pro Europa S.L., Segovia, Spain \\ Correspondence: Ryosuke Iida, School of Agriculture, Meiji University, Higashi-mita 1-1-1, Tama-ku, \\ Kawasaki, Kanagawa 214-8571, Japan. Tel: 81-44-934-7826. E-mail: riid@shikon.meiji.ac.jp
}

Received: September 30, 2017

Accepted: October 31, 2017

Online Published: November 15, 2017

doi:10.5539/jas.v9n12p43

URL: https://doi.org/10.5539/jas.v9n12p43

\begin{abstract}
Our objective was to characterize eating behavior associated with displacement hazard and subsequent performance for pigs were fed in static groups by an electronic sow feeder (ESF). Data included weekly eating records and subsequent farrowing records of 685 pigs. The eating behavior comprised weekly averages of daily feed dispensed (ADFD) and daily total time spent in the feeding stations (TTSF). A displacement female was defined as a pig removed from her group for health reasons. A multivariate model and piecewise exponential models were fitted to the records. Means (inter-quartile ranges) of ADFD and TTSF were $2.4 \mathrm{~kg}(2.1-2.8 \mathrm{~kg})$ and 9.3 $\mathrm{min}$ (7.5-10.8 $\mathrm{min})$, respectively. Gilts had less ADFD than sows during gestational weeks 5-13 $(P<0.05)$, but there was no difference in TTSF between gilts and sows in gestational weeks 5-8 and 11-13 $(P>0.05)$. Also, gilts had higher displacement hazard than parity 2 or higher sows in gestational weeks $8-10(P<0.05)$. Pigs that were entered into the ESF system during summer had less ADFD, and shorter TTSF from gestational weeks 5 to 12 than those entered during the other seasons $(P<0.05)$. The TTSF varied between two genotypes during gestational weeks 5-7 $(P<0.05)$. Also, a higher displacement hazard was associated with less ADFD $(P<0.01)$. A higher hazard of pregnancy loss was associated with shorter TTSF $(P<0.01)$. In conclusion, we recommend that both ADFD and TTSF should be measured in ESF systems to help identity females having problems.
\end{abstract}

Keywords: eating behavior, electronic sow feeder, multivariate analysis, repeated measures

\section{Introduction}

The European swine industry is moving towards group housing because the use of gestation stalls has been banned for mid- and late gestation in all member states of the European Union (EU) since 2013. One of the options which EU producers can choose is an electronic sow feeder (ESF) system (Bench, Rioja-Lang, Hayne, \& Gonyou, 2013a, 2013b; Levis, 2013; Olsson, Andersson, Botermans, Rantzer, \& Svendsen, 2011). The ESF system enables producers to control the amount of feed for each pregnant pig in group housing, and it also records how much feed has been dispensed and how long each pig stays in the feeding station, i.e. its eating behavior. Such eating behavior of mid to late gestation pregnant pigs in ESF systems is not yet well characterized in commercial herds. Records of eating behavior can include weekly average daily feed dispensed (ADFD) and daily total time spent in the feeding stations (TTSF). Appropriate housing of pregnant pigs during mid to late gestation helps ensure they have enough nutrition to develop mammary glands, and adequate placental, fetal and maternal growth (Kraeling \& Webel, 2015). Increased nutrients and energy are needed, especially in late gestation when fetuses are growing rapidly. However, some pregnant pigs do not adapt to the ESF system, and such pigs have to be displaced from the group to a hospital pen or a stall (Bench et al., 2013b; Chapinal et al., 2010a). Despite this, no studies have reported on the displacement hazard of gilts and sows in static groups in commercial herds, nor the association between eating behavior (i.e. ADFD and TTSF) and the displacement hazard or subsequent reproductive performance of sows under the ESF system. Therefore, the objectives of the present study were 1) to characterize the two types of eating behavior (ADFD and TTSF), 2) to assess the displacement hazard for pregnant pigs under an ESF system, and 3) to determine the associations between the pregnant pigs' eating behavior and the displacement hazard, pregnancy loss, and subsequent farrowing and weaning performance in a breeding herd. 


\section{Materials and Methods}

\subsection{Herd with an Electronic Sow Feeder System}

This observational study was conducted on a farrow-to-wean commercial farm housing 500 sows (Segovia, Spain). There was mechanical ventilation in the herd's farrowing, breeding and gestation barns. Every 3 weeks, sows were weaned and then moved to individual stalls for insemination and pregnancy diagnosis. Breeding was conducted using artificial insemination during an estrus period, and a pregnancy was confirmed by real-time ultrasound at 28-35 days after insemination. Lactational and gestational diets were formulated using cereals (barley, wheat and corn) and soybean meal. Replacement gilts in the herds were purchased from the three breeding companies: PIC (PIC España, S. Cugat del Vallés, ES), ACMC (Pure Pig Genetics Ltd, Driffield, UK) and DanBred (DanAvl, Copenhagen, Denmark).

After pregnancy confirmation, pregnant pigs were placed into the ESF system. The group housing pens consist of a $50 \%$ concrete slatted floor and no bedding. The ESF system (GERIONTE, Salamanca, Spain) has four feeding stations for pregnant gilts and sows and was installed in 2014. Under the ESF system, a radio frequency identification (RFID) is attached to each pregnant pig. Each pregnant gilt or sow in the ESF receives 1.8-2.5 kg of feed each day depending on their body condition. Then, for the last 3 weeks of gestation, the amount of feed is increased to 2.0-3.0 kg per day. The dispensed feed amount is estimated on a volumetric basis, with a calibration between volumetric and actual feed weights performed every month. The size of the static groups was approximately 60 sows per group, including both gilts and sows, with a space allowance of $2 \mathrm{~m}^{2}$ per pig.

\subsection{Data and Exclusion Criteria}

Eating records for females that entered the ESF system between January 2014 and October 2015 were extracted from the system, including data on daily feed dispensed and daily total time spent in the feeding stations. The initial dataset contained 100,724 daily eating records in 1,568 pregnancy records of 688 female pigs.

Eating records of pigs' first and last days in the ESF system were excluded (3,132 records). Records on or after the date of pregnancy loss were also excluded (42 records). Daily records showing zero kg feed dispensed were considered as missing records (9,949 records; $10.2 \%$ of 97,550 records), and these were also excluded because ADFD and TTSF would be underestimated. Further records were excluded, if either gestational days at entry was greater than 76 days ( 7 records), or if total time in the system was greater than 79 days (3,048 records). Finally, records showing daily total time spent in the feeding station was 18 min (the mean $+3 \mathrm{SD}$ ) or longer were considered as extreme and excluded (511 records). Hence, the final dataset included 84,035 daily eating records in 1,513 pregnancy records of 685 females. Additionally, the 14,322 records for both ADFD and TTSF were calculated from the daily eating records. These eating records were coordinated with respective reproductive performance data from the PigCHAMP recording system.

Records used in the analysis of eating behaviour were restricted to weeks 5-15 of gestation because there were only 61 records for weeks 3, 4 and 16 of gestation. Also, records of pregnancy loss females were not used in the analysis of displacement hazard.

\subsection{Definitions}

A gilt is defined as a female pig that has entered a herd but has not yet farrowed, and a sow is a female pig that has farrowed at least once. Parity was defined as the number of farrowing, and the number of parities were retained for female pigs with pregnancy loss. In this study, gestation days and gestation weeks were the respective numbers of days and weeks from the date of entry into the system (day 0 and week 0 ). Also, displaced females were defined as females removed from the group for health reasons.

The following measurements were examined: whether or not a pig had pregnancy loss, whether or not a pig had assistance at farrowing, number of piglets born alive, number of stillborn piglets, number of mummified piglets, number of piglets that died less than 24 hours after farrowing, number of piglets that died 24-48 hours after farrowing, number of piglets that died during lactation and the number of weaned piglets.

\subsection{Categories}

Female pigs were categorized into three parity groups: 0 (gilt), 1 and 2 or higher. Entry months were categorized into four quarterly groups (Jan. to Mar., Apr. to Jun., Jul. to Sept. and Oct. to Dec.). Also, genotypes were grouped into the three groups (A, B and C).

\subsection{Statistical Analysis}

Descriptive statistics were performed using SAS University Edition (SAS Institute Inc., Cary, NC, USA). All the models mentioned below included entry year and a block of feeding station. 


\subsubsection{Models for Eating Behavior and Displacement Hazard}

A multivariate longitudinal model was fitted to the weekly eating records by using the GLIMMIX procedure in order to compare eating behavior for different parities, month of entry into the system and genotype (Gao, Thompson, Xiong, \& Miller, 2006). Response variables were ADFD and TTSF, which were assumed to follow normal distribution. This model included the following variables as fixed effects: gestational week, parity groups nested within gestational week, entry month groups nested within gestational week, and genotype groups nested within gestational week. The main effects of parity, entry month and genotype groups were not included in the model because the main effects were not of interest; our research interest for this specific model was only the effects of these variables within each gestational week. Random female effects were also included in the model to allow two intercept terms (one for ADFD and one for TTSF) to vary randomly across female pigs. A separate set of regression coefficients was fitted for each response variable to examine the correlation between these random effects. Two random intercepts were fitted using a RANDOM statement. Also, another RANDOM statement specified that the variances of measurement errors were different for different response variables by using the GROUP option and RESIDUAL option. The TYPE=AR(1) covariance structure was fitted to the repeated measures data. Weekly eating records of females with pregnancy loss were not used in the analysis of eating behavior (107 records). To check the adequacy of the model assumptions, the normality of the random effects and the residuals were evaluated by visual inspection of the normal-probability plots.

A piecewise exponential model was also fitted to the data by using the GLIMMIX procedure in order to estimate displacement hazards for each parity group in each gestational week (Allison, 2010). Parity, entry month and genotype groups were added to the model. Also, the baseline hazard was fitted by a step function (Yang \& Goldstein, 2003). Furthermore, ADFD and TTSF were added separately to different versions of this model as a time-varying variable to examine the association between the respective two types of eating behavior and displacement hazards.

A two-step testing procedure was implemented to test mean differences between groups in each gestational week. In the first step, a global test was performed for the null hypothesis that the expected means of all groups were equal. If this global null hypothesis could be rejected, then in the second step, all pairwise multiple comparisons were made using the Tukey-Kramer method. All significance levels were set at 0.05 .

\subsubsection{Matched Case-Control Study}

A matched case-control study was designed to examine the associations between either ADFD or TTSF and subsequent farrowing performance. One to two case-control matchings were performed to minimize confounding by randomly selecting controls using the SURVEYSELECT procedure (Diseker \& Permanente, 2004). The case and control groups for the number of piglets born alive, the number of piglets that died during lactation and the number of weaned piglets were categorized into two groups based on the $75^{\text {th }}$ percentile of the respective performances (Table 1). The 10 case groups selected were groups of female pigs that experienced pregnancy loss, sows with assisted farrowing, sows with 14 or more piglets born alive, sows with one or more stillborn piglets, sows with one or more mummified piglets, sows with one or more piglets that died less than 24 hours after farrowing, sows with one or more piglets that died 24-48 hours after farrowing, sows with 3 or more piglets that died during lactation and sows with 11 or more weaned piglets. The control groups were matched to the case groups based on parity, entry month and genotype group.

Two piecewise exponential models were fitted to the hazard of pregnancy loss. The ADFD and TTSF were treated as time-varying variables. Parity, entry month and genotype group were also added to the model. Records for pigs displaced from the ESF system were treated as censorings, although records for pigs with pregnancy loss less than one week after displacement were treated as an event. Thus, 24 pregnancy loss records were analyzed as events.

Reverse temporal models (multivariate longitudinal models) were applied using GLIMMIX procedure (Chen, Ferguson, Meeker, McElrath, \& Mukherjee, 2015) to contrast trajectories of eating behavior in matched-pair groups for farrowing and weaning performance. This model included the following variables as fixed effects: gestational week, matched-pair groups nested within gestational week, parity, entry month and genotype group. A separate set of regression coefficients was fitted for each eating behavior. A two-step testing procedure was implemented to test mean differences between cases and controls during the time the pigs were in the ESF system. In the first step, a global test was performed for the null hypothesis that all the differences between the groups are zero in each gestational week. If this null hypothesis was rejected, then in the second step, the differences were checked for each gestational week by using pairwise comparisons. 


\section{Results}

Descriptive statistics for measurements are shown in Table 1. Means (inter-quartile ranges) of ADFD and TTSF were $2.4 \mathrm{~kg}(2.1-2.8 \mathrm{~kg})$ and $9.3 \mathrm{~min}(7.5-10.8 \mathrm{~min})$, respectively.

Table 1. Descriptive statistics for measurements of pigs under an ESF system and their farrowing performance

\begin{tabular}{|c|c|c|c|c|}
\hline Measurements & $\mathrm{N}$ & Mean \pm SE & Median & IQR \\
\hline \multicolumn{5}{|l|}{ Weekly eating records } \\
\hline Average daily feed dispensed, $\mathrm{kg}$ & 14,322 & $2.4 \pm 0.003$ & 2.2 & $2.1-2.8$ \\
\hline Average daily total time spent in the feeding stations, min & 14,322 & $9.3 \pm 0.02$ & 9.6 & $7.5-10.8$ \\
\hline \multicolumn{5}{|l|}{ Pregnancy records } \\
\hline Number of parities & 1,513 & $2.3 \pm 0.05$ & 2.0 & $1-4$ \\
\hline Gestational days at entry into the system & 1,513 & $43.4 \pm 0.26$ & 38.0 & $36-54$ \\
\hline Gestational days at exit from the system & 1,513 & $104.7 \pm 0.28$ & 108.0 & $106-108$ \\
\hline Total days fed in the system & 1,513 & $61.3 \pm 0.38$ & 69.0 & $51-71$ \\
\hline Pregnancy loss, $\%$ & 1,513 & $1.8 \pm 0.34$ & - & - \\
\hline Days from entry into the system to pregnancy loss & 27 & $28.3 \pm 4.42$ & 22.0 & $6-47$ \\
\hline Assisted farrowing, $\%$ & 1,486 & $5.5 \pm 0.59$ & - & - \\
\hline Number of piglets born alive & 1,486 & $12.1 \pm 0.08$ & 12.0 & $11-14$ \\
\hline Number of stillborn piglets & 1,486 & $0.6 \pm 0.03$ & 0.0 & $0-1$ \\
\hline Number of mummified piglets & 1,486 & $0.2 \pm 0.02$ & 0.0 & $0-0$ \\
\hline Number of piglets dying $<24$ hours after farrowing & 1,486 & $0.6 \pm 0.03$ & 0.0 & $0-1$ \\
\hline Number of piglets dying $24-48$ hours after farrowing & 1,486 & $0.2 \pm 0.02$ & 0.0 & $0-0$ \\
\hline Number of piglets dying during lactation & 1,486 & $1.7 \pm 0.05$ & 1.0 & $0-3$ \\
\hline Number of weaned piglets ${ }^{a}$ & 1,430 & $10.1 \pm 0.07$ & 10.0 & $9-11$ \\
\hline
\end{tabular}

Note. ESF: electronic sow feeder; SE: standard error; IQR: inter-quartile range; ${ }^{\mathrm{a}} 56$ records were regarded as missing records.

Both ADFD and TTSF were associated with parity, entry month and genotype. Figure 1 shows the eating behaviors of the three parity groups across gestational weeks. There were significant differences in ADFD between parity groups throughout the period in the ESF system $(P<0.01)$. For example, gilts had $0.13-0.24 \mathrm{~kg}$ less ADFD than sows during weeks $5-13$ of gestation $(P<0.05)$. However, there were only significant differences between parity groups in TTSF during weeks $9-10$ and $14-15$ of gestation $(P \leq 0.03)$; there were no differences in weeks 5-8 and 11-13 of gestation $(P>0.05)$. Also, there were no differences between parity 1 and parity 2 or higher sows for ADFD in weeks 5-7 and 10-13 of gestation, nor for TTSF during weeks 5-13 of gestation $(P>0.05)$. 

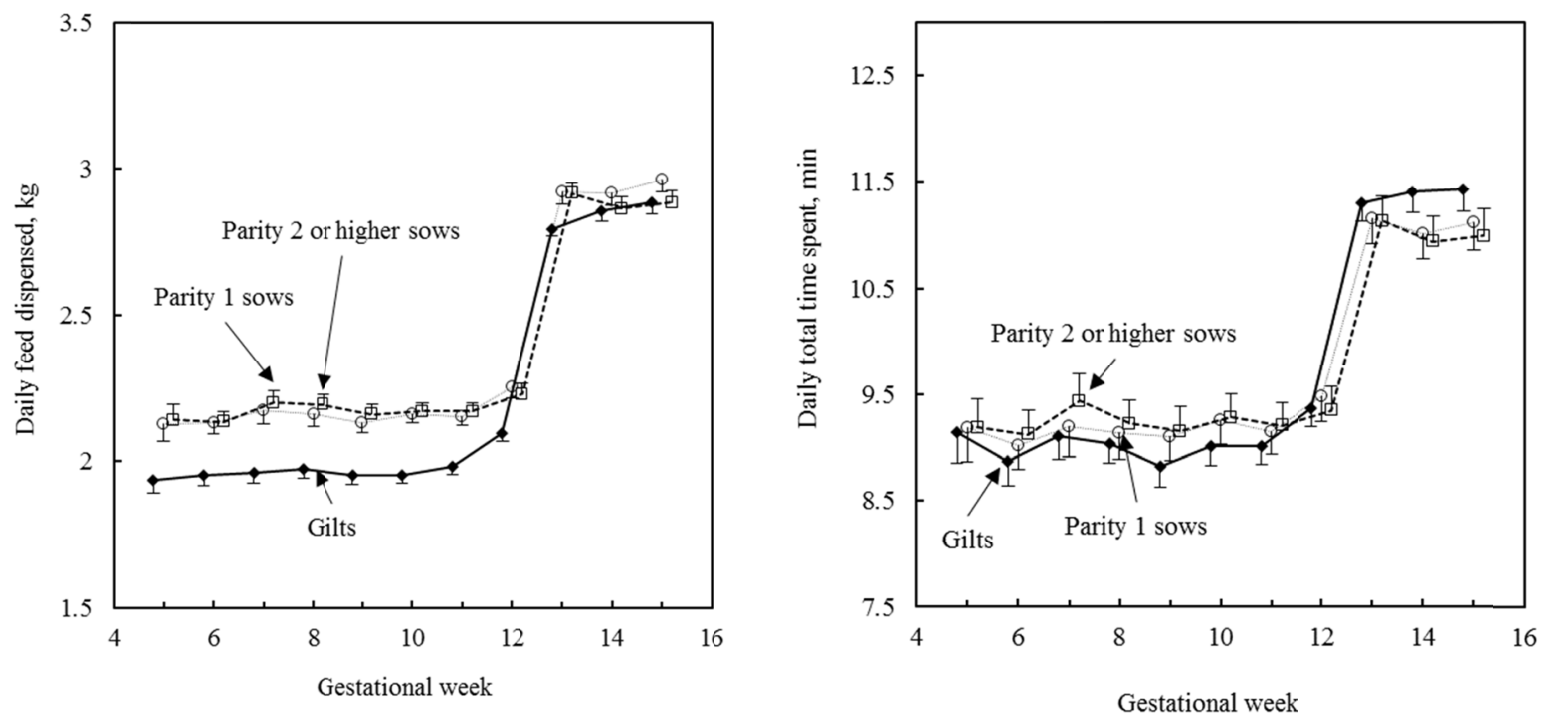

Figure 1. Daily feed dispensed in the electronic sow feeder (ESF) system and daily total time spent in the feeding stations for different parity groups

Note. Means and $95 \%$ confidence intervals were estimated by using the model.

Figure 2 shows the eating behavior of the entry month groups across gestational weeks. There were significant differences in both ADFD and TTSF between seasons throughout the time in the ESF system $(P<0.01)$. For example, pigs that were entered during summer had 0.12-0.28 kg less ADFD, and had 0.28-2.71 min shorter TTSF during weeks 5 to 12 of gestation than those entered during the other seasons $(P<0.05)$.
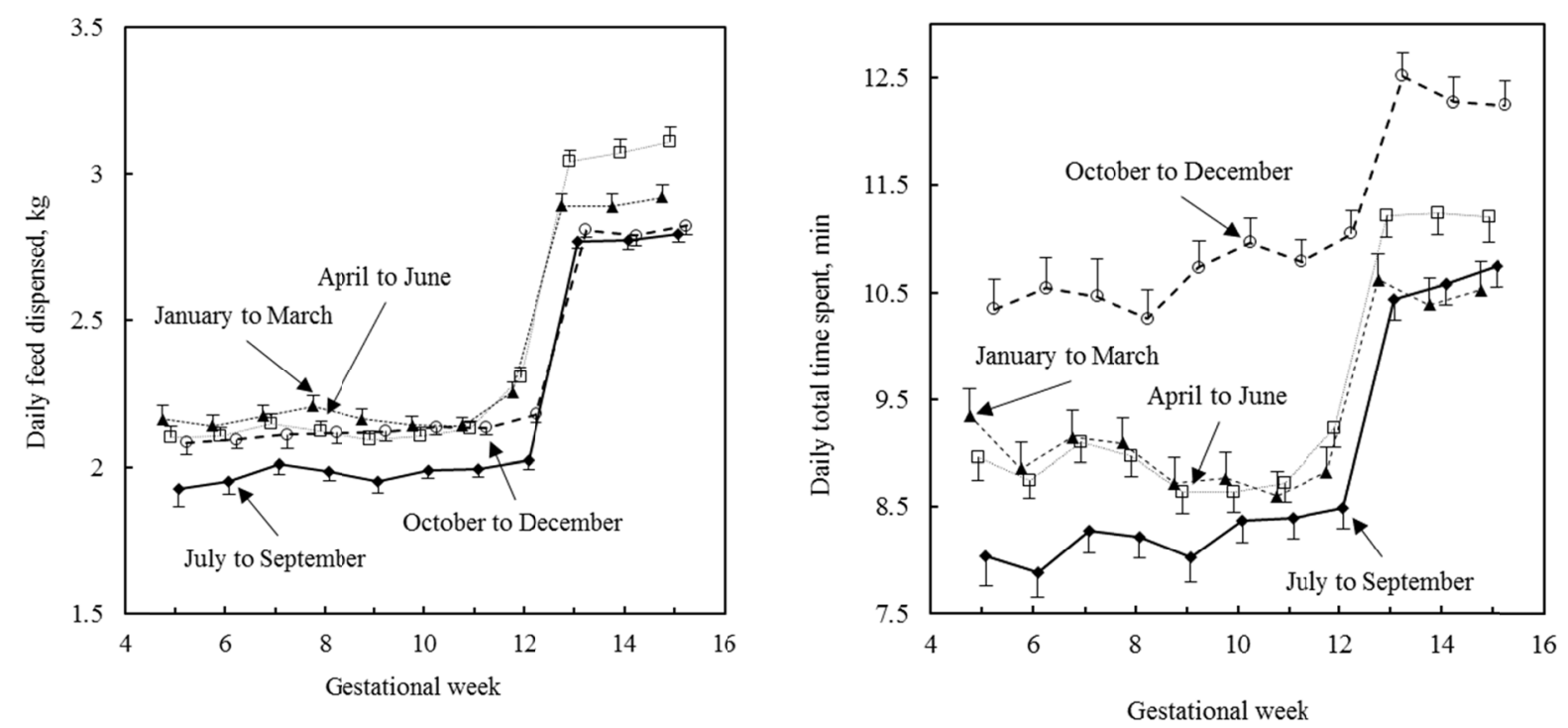

Figure 2. Daily feed dispensed in the electronic sow feeder system (ESF) and daily total time spent in the feeding stations for different seasonal groups

Note. Means and $95 \%$ confidence intervals were estimated by using the model.

Figure 3 shows the eating behavior of genotype groups across gestational weeks. There were significant differences in ADFD between genotypes in weeks 8-11 and 13 of gestation $(P<0.05)$. Also, there were significant differences in TTSF between genotypes in weeks $5-8,10-11$ and 13 of gestation $(P \leq 0.03)$. For 
example, genotype A pigs had 1.02-1.34 min shorter TTSF than genotype B pigs in weeks 7-8 of gestation $(P<$ $0.05)$. However, during this period there was no such difference in ADFD between genotypes A and B pigs $(P>$ 0.05). Also, even though genotype A pigs had 0.43-0.90 min shorter TTSF than genotype C pigs during weeks 5-7 of gestation $(P<0.05)$ there was no similar difference in ADFD at the same time between these genotypes $(P>0.05)$. Additionally, in the analysis of eating behavior, the model estimated that the correlation between ADFD and TTSF within a female pig was 0.68 .
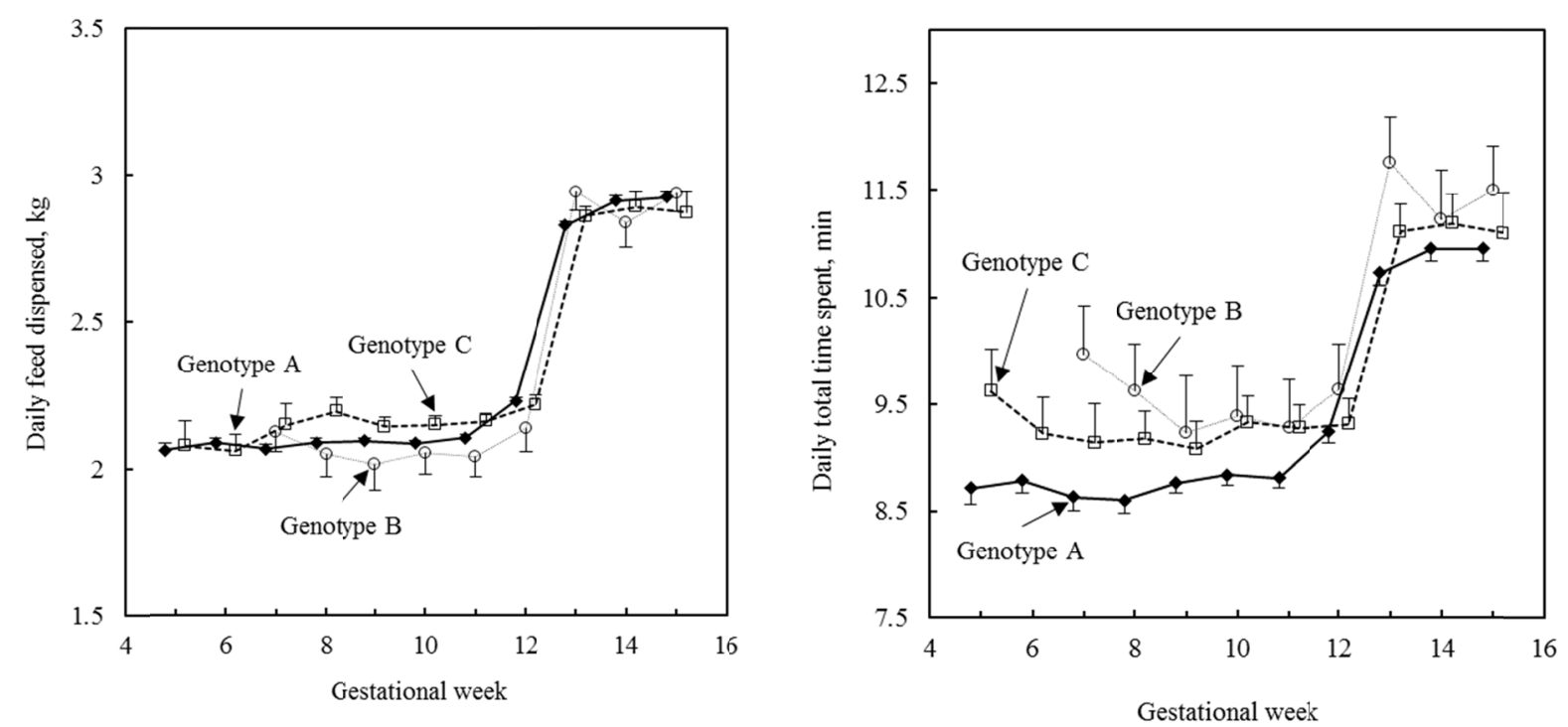

Figure 3. Daily feed dispensed in the electronic sow feeder system and daily total time spent in the feeding stations for different genotype groups

Note. Means and $95 \%$ confidence intervals were estimated by using the model.

The frequency distribution (\%) of displaced females at different weeks of gestation by the 3 parity groups are shown in Figure 4. The percentages of records for gilts, parity 1 and parity 2 or higher sows that were displaced from the ESF system up to week 13 of gestation were 10.8, 5.3 and 2.6\%, respectively. Gilts had 6.2-19.1 times higher displacement hazards than parity 2 or higher sows in weeks $8-10$ of gestation $(P<0.01$; Table 2$)$. Furthermore, a higher displacement hazard was associated with less ADFD (hazard ratio and 95\% CI: 0.750 [0.620-0.907]; $P<0.01)$, but not with TTSF (0.981 [0.947-1.016]; $P=0.29)$. Hence, the displacement hazard was estimated to rise by $2.5 \%$ for each $100 \mathrm{~g}$ decrease in ADFD. 


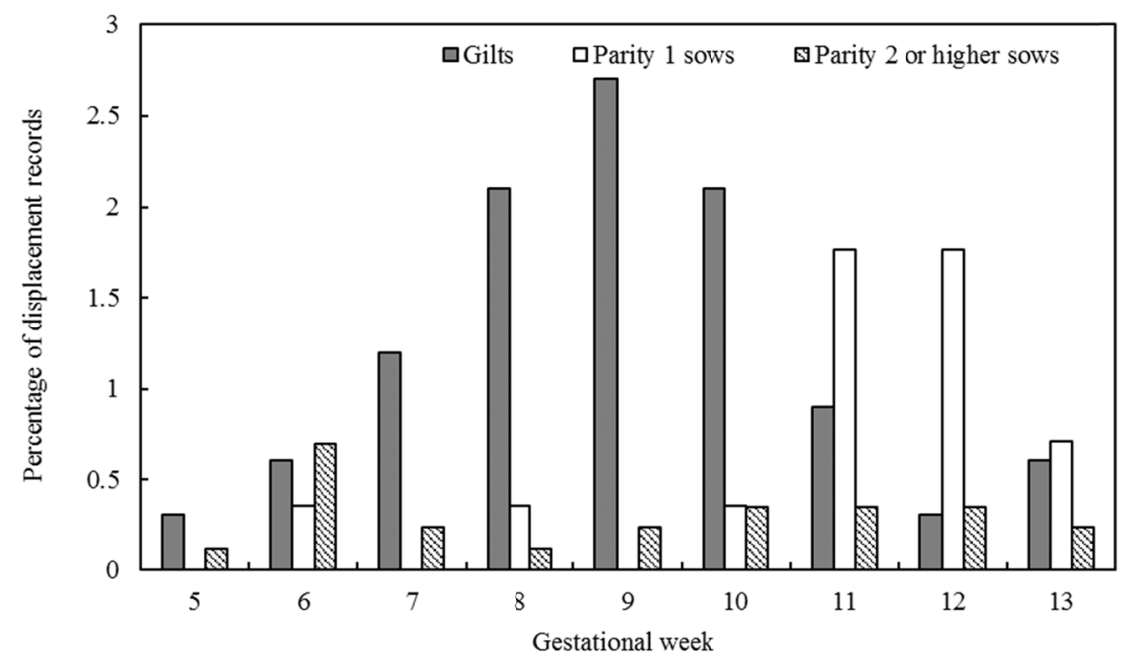

Figure 4. Frequency distributions of 333 pregnancy records of displaced gilts, 283 pregnancy records of displaced parity 1 sows and 870 pregnancy records of displaced parity 2 or higher sows at different weeks of gestation in the ESF system

Note. Records for pigs displaced at or after week 14 of gestation are not shown. Records of pregnancy loss females were not used in this figure.

Table 2. Comparisons between parties for displacement hazards (cases/pig-week) of pigs entered into the ESF system $^{\mathrm{c}}$

\begin{tabular}{llll}
\hline \multirow{2}{*}{ Gestational week } & \multicolumn{1}{c}{ Parity } \\
\cline { 2 - 4 } & Gilts & 1 & 2 or higher \\
\hline 5 & $0.007(0.0010-0.0533)$ & $0.000\left(0.0000-\mathrm{e}^{200.85}\right)$ & $0.002(0.0002-0.0125)$ \\
6 & $0.013(0.0033-0.0526)$ & $0.005(0.0007-0.0371)$ & $0.010(0.0046-0.0233)$ \\
7 & $0.016(0.0061-0.0434)$ & $0.000\left(0.0000-\mathrm{e}^{193.65}\right)$ & $0.003(0.0008-0.0131)$ \\
8 & $0.025(0.0117-0.0517)^{\mathrm{a}}$ & $0.004(0.0006-0.0286)^{\mathrm{ab}}$ & $0.001(0.0002-0.0092)^{\mathrm{b}}$ \\
9 & $0.030(0.0156-0.0582)^{\mathrm{a}}$ & $0.000\left(0.0000-\mathrm{e}^{166.71}\right)^{\mathrm{ab}}$ & $0.003(0.0006-0.0102)^{\mathrm{b}}$ \\
10 & $0.024(0.0114-0.0504)^{\mathrm{a}}$ & $0.004(0.0005-0.0275)^{\mathrm{ab}}$ & $0.004(0.0012-0.0119)^{\mathrm{b}}$ \\
11 & $0.010(0.0034-0.0324)$ & $0.019(0.0080-0.0473)$ & $0.004(0.0012-0.0120)$ \\
12 & $0.004(0.0005-0.0249)$ & $0.020(0.0082-0.0481)$ & $0.004(0.0012-0.0120)$ \\
13 & $0.007(0.0018-0.0282)$ & $0.008(0.0020-0.0323)$ & $0.003(0.0006-0.0103)$ \\
14 & $0.281(0.2200-0.3593)^{\mathrm{a}}$ & $0.088(0.0563-0.1380)^{\mathrm{b}}$ & $0.051(0.0361-0.0725)^{\mathrm{b}}$ \\
15 & $1.938(1.6573-2.2672)$ & $2.120(1.7650-2.5464)$ & $2.165(1.8340-2.5559)$ \\
\hline
\end{tabular}

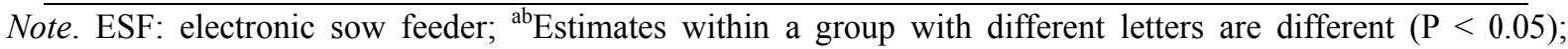
${ }^{c}$ Hazards and $95 \%$ confidence intervals were estimated by using the model.

Pregnancy loss occurred in $1.8 \%$ of pregnancy records between days 3 and 68 after entry into the system (Table 1). Gilts accounted for 20 of 27 pregnancy loss records (74.1\%). A higher pregnancy loss hazard was associated with shorter TTSF $(0.644$ [0.486-0.852]; $P<0.01$; Table 3), but not with the ADFD (hazard ratio and 95\% CI: $0.504[0.079-3.223] ; P=0.47)$. 
Table 3. Estimates of fixed effects included in the proportional hazards model for pregnancy outcome

\begin{tabular}{|c|c|c|c|c|}
\hline \multirow{2}{*}{ Fixed effects ${ }^{\mathrm{a}}$} & \multicolumn{2}{|c|}{ Model 1} & \multicolumn{2}{|c|}{ Model 2} \\
\hline & Estimate $( \pm \mathrm{SE})$ & $P$-value & Estimate $( \pm \mathrm{SE})$ & $P$-value \\
\hline Daily feed dispensed & $-0.686(0.945)$ & 0.47 & - & \\
\hline Daily total time in feeding station & - & & $-0.440(0.143)$ & $<0.01$ \\
\hline Parity & & 0.48 & & 0.51 \\
\hline Gilts & $0.689(0.623)$ & & $0.621(0.629)$ & \\
\hline Parity 1 & $0.774(1.395)$ & & $0.848(1.408)$ & \\
\hline Entry month & & 0.60 & & 0.48 \\
\hline January to March & $0.424(0.934)$ & & $-0.598(1.010)$ & \\
\hline April to June & $1.106(0.822)$ & & $0.850(0.805)$ & \\
\hline July to September & $0.405(0.588)$ & & $-0.139(0.648)$ & \\
\hline Genotype & & 0.30 & & 0.43 \\
\hline A & $1.320(1.048)$ & & $0.975(1.089)$ & \\
\hline $\mathrm{B}$ & $1.648(1.321)$ & & $1.548(1.333)$ & \\
\hline Entry year & & 0.90 & & 0.55 \\
\hline 2014 & $0.13(1.073)$ & & $-0.716(1.193)$ & \\
\hline
\end{tabular}

Note. SE: standard error; ${ }^{a}$ Intercepts and coefficients of the step function and for the feeding station block are not shown in the Table.

Analysis of the trajectories of eating behavior showed that sows having one or more mummified piglets were associated with increased ADFD $(P<0.01)$, but not with TTSF $(P=0.16$; Table 4$)$. Sows with one or more mummified piglets had 0.04-0.05 kg more ADFD than those with no mummified piglets in weeks 11 and 15 of gestation $(P<0.05)$. However, there were no associations between such eating behavior and any of the other farrowing and weaning performance measures; sows having farrowing assistance $(P \geq 0.45)$, sows having 14 or more piglets born alive $(P \geq 0.72)$, sows having one or more stillborn piglets $(P \geq 0.49)$, sows having one or more piglets that died less than 24 hours after farrowing $(P \geq 0.36)$, sows having one or more piglets that died 24-48 hours after farrowing $(P \geq 0.65)$, sows having 3 or more piglets that died during lactation $(P \geq 0.49)$ or sows having 11 or more weaned piglets $(P \geq 0.32)$.

Table 4. P-values in global tests for association between two types of eating behavior and reproductive performance $^{\mathrm{a}}$

\begin{tabular}{lll}
\hline Reproductive performance & Daily feed dispensed & Daily total time staying in the feeding stations \\
\hline Farrowing assistance & 0.48 & 0.45 \\
Piglets born alive & 0.77 & 0.72 \\
Stillborn piglets & 0.69 & 0.49 \\
Mummified piglets & $<0.01$ & 0.16 \\
Piglets dying < 24 hours after farrowing & 0.50 & 0.36 \\
Piglets died dying 24-48 hours after farrowing & 0.66 & 0.65 \\
Piglets dying during lactation & 0.91 & 0.49 \\
Weaned piglets & 0.32 & 0.43 \\
\hline
\end{tabular}

Note. ${ }^{\mathrm{a}}$ Global test's null hypothesis is that all the differences are zero between cases and controls for eating behavior in each gestational week.

\section{Discussion}

To the authors' knowledge, this is the first study that has quantified the weekly hazard of gestating gilts and sows in static groups being displaced from an ESF system. Gilts had a higher risk of displacement than parity 2 or higher sows in weeks $8-10$ of gestation. Also, over $10 \%$ of gilts had been displaced from a group before the expected farrowing date. These results suggest that some gilts cannot adjust to the ESF or cannot get along with other pigs in the ESF system, and have to be removed from the pen. Gilts are generally subordinate to sows, and would be likely to receive more aggression and injuries compared with sows (Levis, 2013). Therefore, gilts 
under ESF systems would have more adaptation failure than sows, and it has been recommended that gilts are housed separately from sows (Levis, 2013; Li, Wang, \& Johnston, 2012). Also, difficulties in adapting to ESF systems could be eased by training the pigs before they are introduced into such a system (Chapinal et al., 2010b).

In the present study, the fact that gilts had lower ADFD than sows, but had similar TTSF can be readily explained by the fact that gilts take longer to eat than sows (Levis, 2013). Therefore, it is necessary to provide gilts with sufficient amounts of feed in the feeding station to maintain their body reserves of protein and fat and to enable them to keep growing. However, there were no differences between parity 1 and parity 2 or higher sows between either type of eating behavior. So, it would not be a problem to house parity 1 sows with parity 2 or higher sows due to their similar eating behavior.

Our study has clearly shown that pregnant pigs were entered into the ESF during summer had the lowest ADFD and TTSF, compared with sows entered in the other seasons. This is most probably due to the increased environmental temperatures during summer leading to a reduction in feed intake by both the gilts and sows (Bergsma \& Hermesch, 2012; Cabezon et al., 2016; Koketsu, Dial, Pettigrew, \& Marsh, 1996; Lewis \& Bunter, 2011). Therefore, it would certainly be recommended that cooling systems (e.g., evaporative cooling systems) are introduced during gestation to help ensure the intake of necessary nutrients and energy by each female.

Genotype differences between the two types of eating behavior suggest that eating speed differs between genotypes. Large variation in eating speed has been reported for dry feed in group housed sows (Boe \& Cronin, 2015). A part of large such variation may be explained by fear or stress related to received aggression in group housing (Kongsted, 2006). Although no research has yet reported any association between eating speed and genotype, it is definitely possible that mixing different genotypes may increase problems in aggression and eating behavior between females.

In this study, a higher displacement hazard was associated with less ADFD. This association could be because female pigs with less ADFD could have more health problems might result in them being removed from the group. Furthermore, our study implies that females that had pregnancy loss have different eating behavior from healthy pregnant pigs. Pregnancy loss females could have been infected by some diseases such as parvovirus or PRRS (Almond, Flowers, Batista, \& D'Allaire, 2006). Therefore, measuring ADFD and TTSF may help producers predict females that have a health problem in the ESF system, or females that are likely to have a problem of pregnancy loss.

There were no direct associations between either type of eating behavior during the 5-15 week mid- to late gestation periods and subsequent farrowing or weaning performance measurements. For example, the number of pigs weaned has been shown to be primarily affected by preweaning mortality, and the number of pigs born alive is mainly associated with insemination timing, management or care during breeding and early gestation phases (Dial, Marsh, Polson, \& Vaillancourt, 1992; Knox, 2016). Also, the number of stillborn piglets is mostly influenced by the total number of pigs born and care in the peri-farrowing phase (Dial et al., 1992; Vanderhaeghe, Dewulf, de Kruif, \& Maes, 2013). The lack of any association between eating behavior and farrowing performance is consistent with the findings of earlier studies that showed no association between pigs born alive and different feeding or energy patterns experimentally imposed in gestation (Ren, Yang, Kim, Menon, \& Baidoo, 2017; Wang et al., 2016). However, our study did indicate that increased ADFD in late gestation was associated with having a mummified piglet at farrowing. Mummified piglets are defined as having died during mid and late gestation after bone mineralization (Almond et al., 2006). However, it is hard to distinguish whether large mummified piglets died in late gestation or healthy piglets died during farrowing. Also, there is no known biological explanation for an association between increased ADFD in late pregnancy and having mummies.

In conclusion, producers could improve the care of gilts and sows in ESF systems if they consider the eating behavior of each pig based on parity, entry month and genotype. Therefore, we recommend that both ADFD and TTSF should be measured in ESF systems as part of daily practice, to help identify females having an eating problem.

There is a limitation with our study, because approximately $10 \%$ of the daily records in pregnant pigs were recorded as $0 \mathrm{~kg}$. We could not confirm whether this $0 \mathrm{~kg}$ means that the pigs did not eat anything on that day or that the EFS systems had mechanical problems. However, even with such a limitation, our study provides unique information on two types of eating behavior in pregnant pigs under an ESF. 


\section{Acknowledgements}

The authors are grateful to Dr. I. McTaggart for his critical review of this manuscript. This work was supported by the Meiji University Giken International Joint Research Project Grant II.

\section{References}

Allison, P. D. (2010). Survival analysis using SAS: A practical guide (2nd ed.). SAS Institute.

Almond, G. W., Flowers, W. L., Batista, L., \& D’Allaire, S. (2006). Diseases of the reproductive system. In B. E. Straw, J. J. Zimmerman, S. D’Allaire, \& D. J. Taylor (Eds.), Diseases of Swine (Vol. 9, pp. 113-147). Ames, IA, USA: Iowa State Univ. Press.

Bench, C. J., Rioja-Lang, F. C., Hayne, S. M., \& Gonyou, H. W. (2013a). Group gestation housing with individual feeding - I: How feeding regime, resource allocation, and genetic factors affect sow welfare. Livest. Sci., 152(2), 208-217. http://dx.doi.org/10.1016/j.livsci.2012.12.021

Bench, C. J., Rioja-Lang, F. C., Hayne, S. M., \& Gonyou, H. W. (2013b). Group gestation sow housing with individual feeding-II: How space allowance, group size and composition, and flooring affect sow welfare. Livest. Sci., 152(2), 218-227. http://dx.doi.org/10.1016/j.livsci.2012.12.020

Bergsma, R., \& Hermesch, S. (2012). Exploring breeding opportunities for reduced thermal sensitivity of feed intake in the lactating sow. J. Anim. Sci., 90(1), 85-98. https://doi.org/10.2527/jas.2011-4021

Boe, K. E., \& Cronin, G. M. (2015). Individual variation in eating speed of dry sows. J. Anim. Sci., 93(4), 1881-1886. https://doi.org/10.2527/jas.2014-8438

Cabezon, F. A., Schinckel, A. P., Richert, B. T., Stewart, K. R., Gandarillas, M., \& Peralta, W. A. (2016). Analysis of lactation feed intakes for sows including data on environmental temperatures and humidity. The Professional Animal Scientist, 32(3), 333-345. http://dx.doi.org/10.15232/pas.2015-01495

Chapinal, N., Ruiz-De-La-Torre, J. L., Cerisuelo, A., Gasa, J., Baucells, M. D., Coma, J., ... Manteca, X. (2010a). Evaluation of welfare and productivity in pregnant sows kept in stalls or in 2 different group housing systems. J. Vet. Behav., 5(2), 82-93. http://dx.doi.org/10.1016/j.jveb.2009.09.046

Chapinal, N., Ruiz-De-La-Torre, J. L., Cerisuelo, A., Gasa, J., Baucells, M. D., \& Manteca, X. (2010b). Aggressive Behavior in Two Different Group-Housing Systems for Pregnant Sows. J. Appl. Anim. Welf. Sci., 13(2), 137-153. https://doi.org/10.1080/10888700903579846

Chen, Y. H., Ferguson, K. K., Meeker, J. D., McElrath, T. F., \& Mukherjee, B. (2015). Statistical methods for modeling repeated measures of maternal environmental exposure biomarkers during pregnancy in association with preterm birth. Environ Health, 14, 9. https://doi.org/10.1186/1476-069x-14-9

Dial, G. D., Marsh, W. E., Polson, D. D., \& Vaillancourt, J. P. (1992). Reproductive failure: differential diagnosis. In A. D. Leman, B. E. Straw, W. L. Mengeling, S. D’Allaire, \& D. J. Taylor (Eds.), Diseases of Swine (Vol. 7, pp. 88-137). Ames, IA, USA: Iowa State Univ. Press.

Diseker, R., \& Permanente, K. (2004). Simplified matched case-control sampling using PROC SURVEYSELECT. Paper presented at the Proceedings of the Twenty-Ninth Annual SAS Users Group International Conference. Retrieved from http://www2.sas.com/proceedings/sugi29/209-29.pdf

Gao, F., Thompson, P., Xiong, C., \& Miller, J. P. (2006). Analyzing multivariate longitudinal data using SAS. Paper presented at the Proceedings of the Thirty-first Annual SAS Users Group International Conference. Retrieved from http:/www2.sas.com/proceedings/sugi31/187-31.pdf

Knox, R. V. (2016). Artificial insemination in pigs today. Theriogenology, 85(1), 83-93. http://dx.doi.org/ 10.1016/j.theriogenology.2015.07.009

Koketsu, Y., Dial, G. D., Pettigrew, J. E., \& Marsh, W. E. (1996). Characterization of feed intake patterns during lactation in commercial swine herds. J. Anim. Sci., 74(6), 1202-1210. https://doi.org/10.2527/1996. $7461202 x$

Kongsted, A. G. (2006). Relation between reproduction performance and indicators of feed intake, fear and social stress in commercial herds with group-housed non-lactating sows. Livest. Sci., 101(1), 46-56. http://dx.doi.org/10.1016/j.livprodsci.2005.09.011

Kraeling, R. R., \& Webel, S. K. (2015). Current strategies for reproductive management of gilts and sows in North America. J. Anim. Sci. Biotechno., 6(1), 3. https://doi.org/10.1186/2049-1891-6-3 
Levis, D. G. (2013). Group Housing Systems: Choices and Designs. National Pork Board Des Moines, IA, USA. Rettieved from http://umanitoba.ca/afs/ncle/pdf/Factsheet-Group_Housing_Systems.Choices_and_Desig ns.pdf

Lewis, C. R. G., \& Bunter, K. L. (2011). Effects of seasonality and ambient temperature on genetic parameters for production and reproductive traits in pigs. Anim. Prod. Sci., 51(7), 615-626. http://dx.doi.org/ 10.1071/AN10265

Li, Y. Z., Wang, L. H., \& Johnston, L. J. (2012). Sorting by parity to reduce aggression toward first-parity sows in group-gestation housing systems. J. Anim. Sci., 90(12), 4514-4522. https://doi.org/10.2527/jas.2011-4869

Olsson, A. C., Andersson, M., Botermans, J., Rantzer, D., \& Svendsen, J. (2011). Animal interaction and response to electronic sow feeding (ESF) in 3 different herds and effects of function settings to increase capacity. Livest. Sci., 137(1), 268-272. http://dx.doi.org/10.1016/j.livsci.2010.10.014

Ren, P., Yang, X. J., Kim, J. S., Menon, D., \& Baidoo, S. K. (2017). Effect of different feeding levels during three short periods of gestation on sow and litter performance over two reproductive cycles. Anim. Reprod. Sci., 177, 42-55. http://dx.doi.org/10.1016/j.anireprosci.2016.12.005

Vanderhaeghe, C., Dewulf, J., de Kruif, A., \& Maes, D. (2013). Non-infectious factors associated with stillbirth in pigs: A review. Anim. Reprod. Sci., 139(1-4), 76-88. https://doi.org/10.1016/j.anireprosci.2013.03.007

Wang, J., Yang, M., Cao, M., Lin, Y., Che, L., Duraipandiyan, V., ... Wu, D. (2016). Moderately increased energy intake during gestation improves body condition of primiparous sows, piglet growth performance, and milk fat and protein output. Livest. Sci., 194, 23-30. http://dx.doi.org/10.1016/j.livsci.2016.09.012

Yang, M., \& Goldstein, H. (2003). Modelling survival data in MLwiN 1.20. Bristol, UK: Univ. of Bristol. Rettieved from http://www.bristol.ac.uk/cmm/media/software/mlwin/downloads/documents/msdm.pdf

\section{Copyrights}

Copyright for this article is retained by the author(s), with first publication rights granted to the journal.

This is an open-access article distributed under the terms and conditions of the Creative Commons Attribution license (http://creativecommons.org/licenses/by/4.0/). 